\title{
COEFFICIENT ESTIMATES FOR STARLIKE FUNCTIONS OF ORDER $\alpha$
}

A. V. BOYD

In [1] MacGregor obtained upper bounds for the moduli of the coefficients of a function $z+\sum_{k+1}^{\infty} a_{n} z^{n}$ which is starlike in the unit disc. The purpose of this note is to extend MacGregor's result to the class of starlike functions of order $\alpha$ introduced by Robertson, [2], and to obtain an improvement on this when $f(z)$ is bounded in the unit disc.

Definition. A function $f(z)$ is said to be starlike of order $\alpha$, $(0 \leqq \alpha<1)$, if it is univalent and $\operatorname{Re}\left\{z f^{\prime}(z) / f(z)\right\} \geqq \alpha$ for $|z|<1$.

TheOREM. If $f(z)=z+\sum_{n=k+1}^{\infty} a_{n} z^{n}$ is starlike of order $\alpha$ then

$$
\left|a_{n}\right| \leqq\left(\begin{array}{c}
(2-2 \alpha) / k+m-1 \\
m
\end{array}\right)
$$

where $m k+1 \leqq n \leqq m k+k, m=1,2,3, \cdots$ If, further, $|f(z)|<1$ for $|z|<1$ then we also have

$$
\sum_{n=p-k+1}^{p}(n+1-2 \alpha)^{2}\left|a_{n}\right|^{2} \leqq 4(1-\alpha)^{2} \quad \text { for } p \geqq k .
$$

\section{LEMMA 1.}

$$
\begin{aligned}
4(1-\alpha)\{1-\alpha & \left.+\sum_{m=1}^{q-1}(m k+1-\alpha)\left[\frac{1}{m !} \prod_{\mu=0}^{m-1}\left(\mu+\frac{2-2 \alpha}{k}\right)\right]^{2}\right\} \\
& =\left\{\frac{k}{(q-1) !} \prod_{\mu=0}^{q-1}\left(\mu+\frac{2-2 \alpha}{k}\right)\right\}^{2} \text { for } q=2,3, \cdots
\end{aligned}
$$

This is easily proved by induction on $q$.

Lemma 2. If $k=1,2, \cdots, q=1,2, \cdots$, and $\alpha<1$ then

$$
(n-1)^{2} \geqq(q k)^{2}(n-\alpha) /(q k+1-\alpha) \text { for } n \geqq q k+1 \text {. }
$$

Proof of Theorem. By the method of $[1]$, if $g(z)=z f^{\prime}(z) / f(z)$ and $h(z)=(g(z)-1) /(g(z)+1-2 \alpha)=b_{k} z^{k}+b_{k+1} z^{k+1}+\cdots \quad$ then $(n-1) a_{n}$ $=2(1-\alpha) b_{n-1}$ for $n=k+1$ to $2 k$, and

$$
\sum_{n=k+1}^{2 k}(n-1)^{2}\left|a_{n}\right|^{2} \leqq 4(1-\alpha)^{2} .
$$

Received by the editors March 16, 1966. 
Also, for some set of constants $d_{n}$,

$$
\begin{aligned}
\sum_{n=k+1}^{p}(n-1) a_{n} z^{n}+\sum_{n=p+1}^{\infty} d_{n} z^{n} & \\
& =h(z)\left\{2(1-\alpha) z+\sum_{n=k+1}^{p-k}(n+1-2 \alpha) a_{n} z^{n}\right\} .
\end{aligned}
$$

Since $|h(z)|<1$ for $|z|<1$ it then follows as in the original paper [3] of Clunie that, for $0 \leqq r<1$,

$$
\sum_{n=k+1}^{p}(n-1)^{2}\left|a_{n}\right|{ }^{2} r^{2 n} \leqq 4(1-\alpha)^{2} r^{2}+\sum_{n=k+1}^{p-k}(n+1-2 \alpha)^{2}\left|a_{n}\right|^{2} r^{2 n} .
$$

Since $a_{1}=1$ and $a_{2}=\cdots=a_{k}=0$ this may be written as

$$
\sum_{n=p-k+1}^{p}(n-1)^{2}\left|a_{n}\right|^{2} r^{2 n} \leqq 4(1-\alpha) \sum_{n=1}^{p-k}(n-\alpha)\left|a_{n}\right|^{2} r^{2 n}
$$

Letting $r$ tend to 1 yields

$$
\sum_{n=p-k+1}^{p}(n-1)^{2}\left|a_{n}\right|^{2}
$$

We next consider

$$
\leqq 4(1-\alpha)\left\{1-\alpha+\sum_{n=k+1}^{p-k}(n-\alpha)\left|a_{n}\right|^{2}\right\}
$$

$$
\sum_{n=m k+1}^{m k+k}(n-1)^{2}\left|a_{n}\right|^{2} \leqq\left\{\frac{k}{(m-1) !} \prod_{\mu=0}^{m-1}\left(\mu+\frac{2-2 \alpha}{k}\right)\right\}^{2}
$$

and

$$
\sum_{n=m k+1}^{m k+k}(n-\alpha)\left|a_{n}\right|^{2} \leqq(m k+1-\alpha)\left\{\frac{1}{m !} \prod_{\mu=0}^{m-1}\left(\mu+\frac{2-2 \alpha}{k}\right)\right\}^{2} .
$$

In the case $m=1$, (A) reduces to equation (i) and (B) follows by an application of Lemma 2 to (A). For $m=q>1,(\mathrm{~A})$ and (B) are proved inductively, as in [1], by applying (B) with $m=1$ to $q-1$ and Lemma 1 to (iii) with $p=(q+1) k$, and Lemma 2 to (A) with $m=q$.

From (A) it follows that, for $m k+1 \leqq n \leqq m k+k$,

$$
\begin{aligned}
\left|a_{n}\right| & \leqq \frac{k}{(n-1)(m-1) !} \prod_{\mu=0}^{m-1}\left(\mu+\frac{2-2 \alpha}{k}\right) \\
& \leqq \frac{1}{m !} \prod_{\mu=0}^{m-1}\left(\mu+\frac{2-2 \alpha}{k}\right) .
\end{aligned}
$$


It should be noted that this result

$$
\left|a_{n}\right| \leqq\left(\begin{array}{c}
(2-2 \alpha) / k+m-1 \\
m
\end{array}\right)
$$

is "sharp" for $n=m k+1,(m=1,2, \cdots)$, for the function $f(z)$ $=z\left(1-z^{k}\right)^{-2(1-\alpha) / k}$; and that when $\alpha=0$ it gives the same bounds $s_{\alpha}^{\prime \prime}$ for the coefficients as Waadeland [4] found in the case of $k$-symmetric univalent functions.

We also have, from (ii), that

$$
\begin{aligned}
\sum_{n=p-k+1}^{p}(n+1-2 \alpha)^{2}\left|a_{n}\right|{ }^{2} r^{2 n} & \leqq 4(1-\alpha) \sum_{n=1}^{p}(n-\alpha)\left|a_{n}\right|{ }^{2} r^{2 n} \\
& \leqq 4(1-\alpha) \sum_{n=1}^{\infty}(n-\alpha)\left|a_{n}\right|^{2} r^{2 n}
\end{aligned}
$$

As on page 232 of [5], the right hand-side does not exceed

$$
\begin{aligned}
& \frac{2(1-\alpha)}{\pi} \int_{0}^{2 \pi}\left\{\operatorname{Re} \frac{z f^{\prime}(z)}{f(z)}-\alpha\right\} d \theta \quad \text { where } z=r e^{i \theta} \\
& =4(1-\alpha)^{2} .
\end{aligned}
$$

It now follows that

$$
\sum_{n=p-k+1}^{p}(n+1-2 \alpha)^{2}\left|a_{n}\right|^{2} \leqq 4(1-\alpha)^{2} \quad \text { for } p \geqq k .
$$

The author would like to thank the referee for his useful comments and his reference to [4].

\section{REFERENCES}

1. T. H. MacGregor, Coefficient estimates for starlike mappings, Michigan Math. J. 10 (1963), 277-281.

2. M. S. Robertson, On the theory of univalent functions, Ann. of Math. 37 (1936), 374-408.

3. J. Clunie, On meromorphic schlicht functions, J. London Math. Soc. 34 (1959), 215-216.

4. H. Waadeland, Über $k$-fach symmetrische, sternförmige, schlichte Abbildungen des Einheitskreises, Math. Scand. 3 (1955), 150-154.

5. C. Pommerenke, On meromorphic starlike functions, Pacific J. Math. 13 (1963), 221-236.

University of the Witwatersrand, Johannesburg, South Africa 